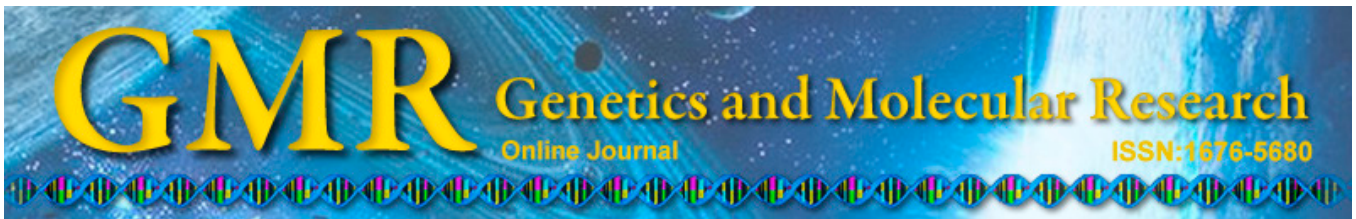

\title{
Screening of differentially expressed genes in pathological scar tissues using expression microarray
}

\author{
L.P. Huang ${ }^{1 *}$, Z. Mao ${ }^{2 *}$, L. Zhang ${ }^{3}$, X.X. Liu ${ }^{1}$, C. Huang and Z.S. Jia ${ }^{1}$ \\ ${ }^{1}$ Department of Physical Therapy, Chinese PLA General Hospital, \\ Beijing, China \\ ${ }^{2}$ Department of Orthopedics, Chinese PLA General Hospital, Beijing, China \\ ${ }^{3}$ Dalian City Hospital of Traditional Chinese Medicine, Dalian, China \\ *These authors contributed equally to this study. \\ Corresponding author: Z.S. Jia \\ E-mail: wangx1301@126.com / jzs1963@163.com
}

Genet. Mol. Res. 14 (3): 10743-10751 (2015)

Received January 7, 2015

Accepted May 25, 2015

Published September 9, 2015

DOI http://dx.doi.org/10.4238/2015.September.9.13

\begin{abstract}
Pathological scar tissues and normal skin tissues were differentiated by screening for differentially expressed genes in pathologic scar tissues via gene expression microarray. The differentially expressed gene data was analyzed by gene ontology and pathway analyses. There were 5001 up- or down-regulated genes in 2-fold differentially expressed genes, 956 up- or down-regulated genes in 5-fold differentially expressed genes, and 114 up- or downregulated genes in 20-fold differentially expressed genes. Therefore, significant differences were observed in the gene expression in pathological scar tissues and normal foreskin tissues. The development of pathological scar tissues has been correlated to changes in multiple genes and pathways, which are believed to form a dynamic network connection.
\end{abstract}

Key words: Pathological scar tissue; Differentially expressed gene; Gene chip 


\section{INTRODUCTION}

Scar tissues are the end result of skin wound healing; however, uncontrolled synthesis and metabolism of skin collagen during the process of healing results in a continuous hyperthyroidism state, which leads to hyperplasia of the collagen fiber. This results in the formation of a pathological scar, which is characterized by a big protrusion out of the skin, an irregular shape, and the formation of red, hard benign masses. The formation of pathological scar is closely related to abnormal gene expression in the pathological scar tissue. In this study, Genechip U133 Plus 2.0 gene expression microarray was used to compare the differential expression of genes in pathological scar and normal skin tissues; this was achieved by screening for differentially expressed genes in both types of tissues, and conducting a preliminary identification of the function of these genes.

\section{MATERIAL AND METHODS}

\section{Clinical data}

The total number of patients was 8 and was divided into two groups: the pathological scar group ( 3 cases) and the normal skin group (5 cases). Pathological scar tissue samples were obtained from patients with pathological scars treated at the Department of Dermatology in the People's Liberation Army (PLA) General Hospital. The scar tissue samples were cut and sent to the Department of Pathology at the PLA General Hospital, for a formal diagnosis of pathological scars. The normal skin tissues were obtained from patients undergoing circumcision at the Department of Urology at PLA General Hospital. Informed consent was obtained from all patients who provided samples (or who were operated on). The case data has been summarized in Table 1 .

Table 1. Patient characteristics.
\begin{tabular}{lllllr}
\hline No. & Gender & Age & Donor site & Diagnosis & Disease history (years) \\
\hline 1 & Male & 35 & Chest & Folliculitis & 1 \\
2 & Male & 41 & Chest & Furuncle & 2 \\
3 & Male & 29 & Chest & After surgery & 1.6 \\
\hline
\end{tabular}

\section{Screening for differentially expressed genes}

The ratio of signal value was used to analyze the chip in order to screen for differentially expressed genes. The Genechip was scanned by laser scanning confocal microscope (OLS4100, Olympus Imaging Co., Ltd, China). The ratio of signal value was analyzed online at http://rana.lbl.gov/EisenSoftware.htm. The differential expression was analyzed online at $\mathrm{http} / / /$ cybert.microarray.ics.uci.edu. Those whose ratio was above 50 times were considered as up-regulation, while those below 50 times were considered as down-regulation. The $t$-test was used for analysis and a value of $\mathrm{P}<0.05$ was considered to be significantly.

\section{Analysis of differentially expressed genes with Gene Ontology (GO) and pathway analyses}

The GO analysis was conducted using the DAVID Bioinformatics Resources 6.7 software platform, provided by the United States National Institute of Allergy and Infectious Diseas- 
es; on the other hand, pathway analyses were performed online at http://david.abcc.ncifcrf.gov/.

\section{RESULTS}

\section{RNA integrity}

The obtained RNA samples gave clear bands when subjected to formaldehyde denaturing gel electrophoresis. The band brightness of $28 \mathrm{~S}: 18 \mathrm{~S}$ rRNA was greater than or close to $1: 1$. The quality of the bands was in line with the basic requirement of the Affymetrix expression microarray system (Figure 1A and B).
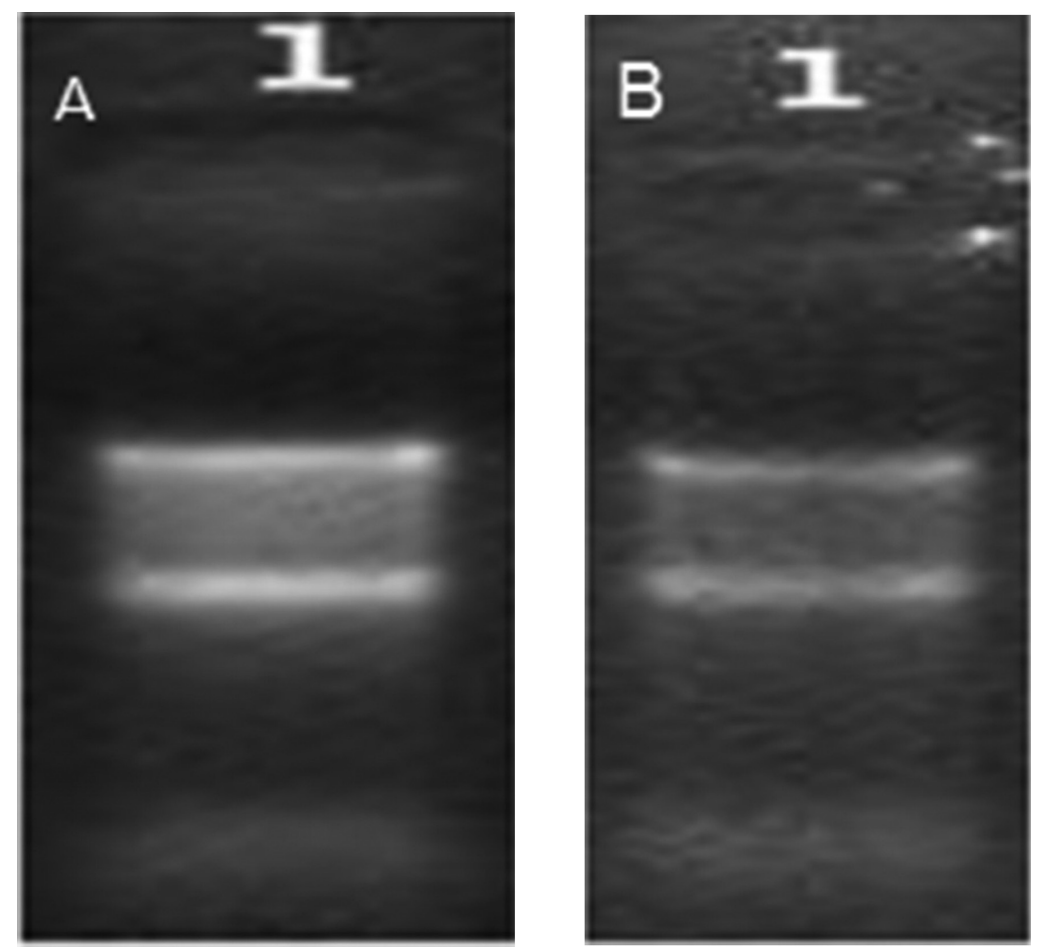

Figure 1. Electrophoresis strip of RNA extracted from normal foreskin tissue (A), and pathological scar tissue (B) from the chest of the patient.

\section{Results of screening for differential expression of genes}

The ratio of signal value was used to screen the whole gene expression profile obtained using the Genechip U133 plus 2.0 microarray. The pathological scar tissue showed differential expression of genes compared to normal human foreskin tissue. There were 5001 up- or down-regulated genes in 2-fold differentially expressed genes (2942 up and 2059 down) and 956 up- or down-regulated genes in 5-fold differentially expressed genes (699 up and 357 down). There were 144 up- or down-regulated genes in 20 -fold differentially expressed genes (89 up and 55 down) and 38 up- or down-regulated genes in 50-fold differentially expressed genes (19 up and 19 down).(Tables 2 and 3). 
Table 2. List of differentially expressed genes in the pathological scar tissue up-regulated by more than 50 times compared to the normal foreskin tissue.

\begin{tabular}{|c|c|c|c|}
\hline Probe Set ID & Ratio & Gene Title & Gene symbol \\
\hline 213790 at & 394.753 & ADAM metallopeptidase domain 12 & $A D A M 12$ \\
\hline $202952^{-} \mathrm{s}$ at & 340.571 & ADAM metallopeptidase domain 12 & $A D A M 12$ \\
\hline 204320 at & 326.908 & Collagen, type XI, alpha 1 & COL11A1 \\
\hline 226777_at & 270.562 & - & - \\
\hline 37892 at & 219.961 & Collagen, type XI, alpha 1 & COL11A1 \\
\hline $22947 \overline{9}$ at & 117.305 & - & - \\
\hline $229802^{-}$at & 94.4577 & - & - \\
\hline $236044^{-}$at & 93.6993 & Phosphatidic acid phosphatase type 2 domain containing $1 \mathrm{~A}$ & PPAPDC1A \\
\hline $229152^{-}$at & 92.2468 & Chromosome 4 open reading frame 7 & C4orf7 \\
\hline 224396 s at & 84.1051 & Asporin & $A S P N$ \\
\hline 204052_s_at & 83.8197 & Secreted frizzled-related protein 4 & SFRP4 \\
\hline 204776 at & 83.508 & Thrombospondin 4 & THBS4 \\
\hline 213909_at & 78.8505 & Leucine rich repeat containing 15 & LRRC15 \\
\hline $226237^{-}$at & 68.9421 & - & - \\
\hline 217428_s_at & 66.1755 & Collagen, type $\mathrm{X}$, alpha 1 & COL10A1 \\
\hline $209840^{-} \mathrm{s}$ at & 58.5571 & Leucine rich repeat neuronal 3 & $L R R N 3$ \\
\hline 231766 s at & 57.0013 & Collagen, type XII, alpha 1 & COL12A1 \\
\hline 206796_at & 56.5772 & WNT1 inducible signaling pathway protein 1 & WISPl \\
\hline $243802^{-}$at & 54.5355 & Dynein, axonemal, heavy chain 12 & DNAH12 \\
\hline 205713 s_at & 47.9473 & Cartilage oligomeric matrix protein & COMP \\
\hline
\end{tabular}

Table 3. List of differentially expressed genes in the pathological scar tissue down-regulated by more than 20 times compared to normal foreskin tissues.

\begin{tabular}{|c|c|c|c|}
\hline Probe Set ID & Ratio & Gene Title & Gene Symbol \\
\hline 207935_s_at & 0.0012 & Keratin 13 & KRT13 \\
\hline $155394 \overline{6}$ at & 0.0024 & Dermicidin & $D C D$ \\
\hline 213240 s at & 0.0038 & Keratin 4 & KRT4 \\
\hline 206378 at & 0.0039 & Secretoglobin, family $2 \mathrm{~A}$, member 2 & $S C G B 2 A 2$ \\
\hline $229477^{-}$at & 0.0068 & Thyroid hormone responsive (SPOT14 homolog, rat) & THRSP \\
\hline 41469 at & 0.0076 & Peptidase inhibitor 3 , skin-derived & PI3 \\
\hline $22947 \overline{6}$ s at & 0.0085 & Thyroid hormone responsive (SPOT14 homolog, rat) & THRSP \\
\hline $203691^{-}$at & 0.0087 & Peptidase inhibitor 3 , skin-derived & $P I 3$ \\
\hline 205549 at & 0.0089 & Purkinje cell protein 4 & PCP4 \\
\hline $155884 \overline{6}$ at & 0.0097 & Pancreatic lipase-related protein 3 & PNLIPRP3 \\
\hline 234513 at & 0.0139 & Elongation of very long chain fatty acids (FEN1/Elo2, SUR4/Elo3, yeast)-like 3 & ELOVL3 \\
\hline $219554^{-}$at & 0.0149 & $\mathrm{Rh}$ family, C glycoprotein & $R H C G$ \\
\hline 207430 s_at & 0.0178 & Microseminoprotein, beta- & $M S M B$ \\
\hline 205029 s at & 0.0186 & Fatty acid binding protein 7 , brain & FABP7 \\
\hline 212531_at & 0.0189 & Lipocalin 2 & $L C N 2$ \\
\hline $205783^{-}$at & 0.0193 & Kallikrein-related peptidase 13 & KLK13 \\
\hline 210297 s_at & 0.0193 & Microseminoprotein, beta- & $M S M B$ \\
\hline 207802 at & 0.0199 & Cysteine-rich secretory protein 3 & CRISP3 \\
\hline 205030 at & 0.02 & Fatty acid binding protein 7 , brain & FABP7 \\
\hline $214240^{-}$at & 0.0213 & Galanin prepropeptide & $G A L$ \\
\hline
\end{tabular}

\section{Results of GO analysis}

\section{GO analysis for molecular function}

The up-regulated differentially expressed genes were associated with the following specific molecular functions: interactions with protein and metal ions, composition of extracellular matrix, peptidase activity, integration of binding protein, transcription factor activity (Table 4 lists the top 10 results by $\mathrm{P}$ value).

On the other hand, down-regulated differentially expressed genes were mainly related to the following molecular functions: enzyme activity, interactions between and movement of ions, and ion channel activity (Table 5 lists the top 10 results based on P value). 
Table 4. Results of GO analysis of up-regulated differentially expressed genes affecting molecular function.

\begin{tabular}{lcr}
\hline GO molecular function & Count & P value \\
\hline GO:0005515 protein binding & 110 & $5.55 \mathrm{E}-92$ \\
GO:0005509 calcium ion binding & 50 & $1.13 \mathrm{E}-68$ \\
GO:0005201 extracellular matrix structural constituent & 18 & $7.90 \mathrm{E}-37$ \\
GO:0008233 peptidase activity & 22 & $2.13 \mathrm{E}-27$ \\
GO:0004222 metalloendopeptidase activity & 14 & $9.11 \mathrm{E}-26$ \\
GO:0005178 integrin binding & 12 & $2.17 \mathrm{E}-25$ \\
GO:0008270 zinc ion binding & 30 & $3.64 \mathrm{E}-23$ \\
GO:0003700 transcription factor activity & 22 & $9.57 \mathrm{E}-23$ \\
GO:0046872 metal ion binding & 35 & $4.97 \mathrm{E}-21$ \\
GO:0003823 antigen binding & 13 & $1.33 \mathrm{E}-20$ \\
\hline
\end{tabular}

Table 5. Results of GO analysis of down-regulated differentially expressed genes affecting molecular function.

\begin{tabular}{lcc}
\hline GO molecular function & Count & P value \\
\hline GO:0005515 protein binding & 53 & $2.48 \mathrm{E}-34$ \\
GO:0004252 serine-type endopeptidase activity & 14 & $6.56 \mathrm{E}-25$ \\
GO:0016491 oxidoreductase activity & 19 & $7.18 \mathrm{E}-24$ \\
GO:0004867 serine-type endopeptidase inhibitor activity & 12 & $1.23 \mathrm{E}-23$ \\
GO:0008233 peptidase activity & 17 & $1.00 \mathrm{E}-21$ \\
GO:0005509 calcium ion binding & 18 & $9.79 \mathrm{E}-20$ \\
GO:0020037 heme binding & 10 & $7.82 \mathrm{E}-18$ \\
GO:0046872 metal ion binding & 27 & $6.60 \mathrm{E}-17$ \\
GO:0005198 structural molecule activity & 14 & $2.19 \mathrm{E}-16$ \\
GO:0042803 protein homodimerization activity & 11 & $2.22 \mathrm{E}-16$ \\
\hline
\end{tabular}

\section{GO analysis for biological process}

The up-regulated differentially expressed genes were mainly associated with the following biological processes: cell adhesion, formation of collagen fibers, decomposition of collagen, cell differentiation, cell signal transduction, cell hypoxia response, cell matrix adhesion, integrin-mediated signal pathway, and nerve bone development (Table 6 lists the top 10 results by $\mathrm{P}$ value).

The down-regulated differentially expressed genes were related to other biological processes, such as cellular redox reaction, cell differentiation, lipid metabolism and synthesis, and inflammatory reaction (Table 7 lists the top 10 results by $\mathrm{P}$ value).

Table 6. Results of GO analysis of up-regulated differentially expressed genes affecting the biological process.

\begin{tabular}{lcc}
\hline GO biological process & Count & P value \\
\hline GO:0007155 cell adhesion & 52 & $2.61 \mathrm{E}-76$ \\
GO:0007275 development & 38 & $5.35 \mathrm{E}-32$ \\
GO:0006955 immune response & 26 & $3.72 \mathrm{E}-29$ \\
GO:0030199 collagen fibril organization & 11 & $3.45 \mathrm{E}-27$ \\
GO:0006508 proteolysis & 23 & $4.27 \mathrm{E}-23$ \\
GO:0006355 regulation of transcription, DNA-dependent & 28 & $9.79 \mathrm{E}-21$ \\
GO:0001501 skeletal development & 13 & $7.26 \mathrm{E}-18$ \\
GO:0055114 oxidation reduction & 16 & $1.13 \mathrm{E}-17$ \\
GO:0007399 nervous system development & 14 & $4.67 \mathrm{E}-13$ \\
GO:0030154 cell differentiation & 16 & $1.70 \mathrm{E}-12$ \\
\hline
\end{tabular}


Table 7. Results of GO analysis of down-regulated differentially expressed genes affecting the biological process.

\begin{tabular}{lrr}
\hline GO biological process & Count & P value \\
\hline GO:0055114 oxidation reduction & 26 & $1.10 \mathrm{E}-36$ \\
GO:0006508 proteolysis & 21 & $5.09 \mathrm{E}-23$ \\
GO:0008544 epidermis development & 11 & $1.07 \mathrm{E}-18$ \\
GO:0030216 keratinocyte differentiation & 8 & $7.63 \mathrm{E}-18$ \\
GO:0031424 keratinization & 8 & $1.18 \mathrm{E}-17$ \\
GO:0030855 epithelial cell differentiation & 6 & $4.29 \mathrm{E}-12$ \\
GO:0006629 lipid metabolism & 10 & $9.89 \mathrm{E}-10$ \\
GO:0019370 leukotriene biosynthesis & 4 & $1.41 \mathrm{E}-09$ \\
GO:0016338 calcium-independent cell-cell adhesion & 4 & $2.66 \mathrm{E}-09$ \\
GO:0001666 response to hypoxia & 5 & $4.36 \mathrm{E}-09$ \\
\hline
\end{tabular}

\section{GO analysis for cell components}

The up-regulated differentially expressed genes were associated with the extracellular domain, extracellular matrix, dynamic cell membrane protein, extracellular space, endoplasmic reticulum, Golgi body, basement membrane, collagen, type $\mathrm{V}$ collagen, platelet alpha granule, and other such cellular components (Table 7 lists the top 10 results by P value).

On the other hand, the down-regulated differentially expressed genes were mainly related to the extracellular matrix, cell membrane, endoplasmic reticulum, plasma membrane, cornified envelope, and intermediate fiber cellular components (Table 8 lists the top 10 results by $\mathrm{P}$ value).

\section{Results of pathway analysis}

The DAVID software platform was used to analyze the 956 genes that were differentially expressed by $>5$ times (599, up-regulation; 357, down-regulation); 61 pathways were analyzed in the KEGG map, out of which 23 were associated with the up-regulated differentially expressed genes and 38 were related to the down-regulated differentially expressed genes (Table 8 and 9 list the top 10 results by $\mathrm{P}$ value).

Table 8. Results of GO analysis of down-regulated differentially expressed genes affecting the cellular composition.

\begin{tabular}{lcr}
\hline GO cellular component & Count & P value \\
\hline GO:0005576 extracellular region & 72 & $4.45 \mathrm{E}-95$ \\
GO:0016020 membrane & 62 & $6.37 \mathrm{E}-45$ \\
GO:0005737 cytoplasm & 59 & $6.28 \mathrm{E}-42$ \\
GO:0016021 integral to membrane & $53.03 \mathrm{E}-41$ \\
GO:0005783 endoplasmic reticulum & 27 & $6.07 \mathrm{E}-34$ \\
GO:0005886 plasma membrane & 38 & $1.62 \mathrm{E}-30$ \\
GO:0005789 endoplasmic reticulum membrane & 21 & $7.43 \mathrm{E}-29$ \\
GO:0005615 extracellular space & 20 & $6.54 \mathrm{E}-27$ \\
GO:0005887 integral to plasma membrane & 23 & $7.19 \mathrm{E}-25$ \\
GO:0001533 cornified envelope & 9 & $1.60 \mathrm{E}-23$ \\
\hline
\end{tabular}


Table 9. Biological pathways affected by the down-regulated differentially expressed genes.

\begin{tabular}{|c|c|c|}
\hline Pathway & P value & Genes \\
\hline Drug metabolism - cytochrome P450 & $9.12 \mathrm{E}-07$ & $\begin{array}{l}\text { MGST1; MGST1; MGST1; MAOA; FMO5; UGT2A1; MAOA; CYP } 3 A 5 \text {; } \\
\text { ALDH3A1; CYP } 3 A 5\end{array}$ \\
\hline Arachidonic acid metabolism & $5.92 \mathrm{E}-06$ & $A L O X 15 B ; A L O X 15 B ; C Y P 2 J 2 ; G P X 3 ; A L O X 12 ; A L O X 12 B ; G P X 3$ \\
\hline Urea cycle and metabolism of amino groups & 7.33E-06 & ARG2;ARG1;MAOA;MAOA; ALDH3A1 \\
\hline Androgen and estrogen metabolism & $5.03 \mathrm{E}-05$ & $\begin{array}{l}\text { SULT1E1; SULT1E1; HSD17B2; } \\
\text { HSD11B1; UGT2A1 }\end{array}$ \\
\hline Tyrosine metabolism & $5.98 \mathrm{E}-05$ & PNPLA3; MAOA; MAOA; ALDH3A1; TYR \\
\hline Phenylalanine metabolism & $1.28 \mathrm{E}-04$ & PNPLA3; MAOA; MAOA; ALDH3A1 \\
\hline Retinol metabolism & $2.13 \mathrm{E}-04$ & $\begin{array}{l}\text { DGAT2; DGAT2; UGT2A1; CYP } 3 A 5 \\
\text { CYP } 3 A 5 ; \text { DHRS9 }\end{array}$ \\
\hline Biosynthesis of steroids & $2.66 \mathrm{E}-04$ & TM7SF2; DHCR7; SC4MOL \\
\hline Metabolism of xenobiotics by cytochrome P450 & $2.83 \mathrm{E}-04$ & $\begin{array}{l}\text { MGST1; MGST1; MGST1; UGT2A1; CYP } 3 A 5 ; \\
\text { ALDH3A1; CYP } 3 A 5\end{array}$ \\
\hline Histidine metabolism & $3.62 \mathrm{E}-04$ & $H A L ; M A O A ; M A O A ; A L D H 3 A 1$ \\
\hline Tight junction & $3.83 \mathrm{E}-04$ & $\begin{array}{l}\text { MYH11; MYH11; MYH11; CLDN17; CLDN8; } \\
\text { CLDN11; CLDN4 }\end{array}$ \\
\hline Sphingolipid metabolism & 7.17E-04 & $S G P P 2 ; A C E R 1 ; D E G S 2$ \\
\hline
\end{tabular}

\section{DISCUSSION}

A majority of the research conducted into scar formation-related genes thus far has focused on the identification of the role played by individual genes. However, previous research has also shown that the formation of scars during skin wound healing is a result of a complicated, dynamic network of gene expression and metabolic regulation (Chen et al., 2012; Ray et al., 2013). Therefore, research into individual genes has some limitations (Wang, 2007) compared to fast and accurate gene analysis methods, which are used to screen for the genes that are associated with the formation of pathological scars. An effective method to explain the pathogenesis of hypertrophic scars is to explore the relationship between genes and pathways. Gene chip technology can be used to screen a large number of genes in parallel (Ayari and Bricca, 2013; Yoshida and Ishibashi, 2013). Gene expression profiling enables researchers to obtain a vast amount of information on related gene expression through tissue analysis (using a small amount of sample). Analysis of the expression spectrum of gene chips has been widely applied to study the differential expression of genes in normal and carcinoma tissues; in fact, this has facilitated the discovery of many important and relevant characteristics of carcinoma tissues. (Simpson et al., 2008; Shi et al., 2009). In this study, the GeneChip ${ }^{\circledR}$ Human Genome U133 Plus 2.0 expression microarray was used to analyze and screen for differentially expressed genes in pathological scar and normal foreskin tissues. Gene chips can be used to analyze a large number of gene expressions simultaneously, producing extensive experimental data; however, the up- and down-regulated genes cannot be identified in a majority of the cases. Gene expression data is linked to the molecular function or known signaling pathways, which facilitates the interpretation of the microarray data, and may help identify mechanisms underlying changes in gene expression (Zhao and Shao, 2009).

In this study, we utilized the GO and Pathway gene function analysis methods in order to analyze the differential expression of genes. The GO database contains 17,348 annotated genes; these are divided into three categories based on the role played by these genes in molecular function, biological processes, and cellular components. Analysis of the obtained microarray data can help researchers identify the common GO function branch that can accommodate a genetic change, thereby identifying the gene changes that could affect the bio- 
logical function. Pathway analysis is a widely used microarray data analysis method used to identify changes in gene function. Pathway analysis data has been used to study the changes in biological pathways affected by differential gene expression; this method uses individual gene expression data, and therefore differs from the GO classification. In this method, the list of differentially expressed genes is inputted, the pathway analysis software is imported, the pathways that are affected by the differential expression of these genes is identified, and the pathway that is significantly affected by the changes in gene expression is calculated. In this study, the pathway analysis was conducted using information extracted from the KEGG database. Each of the biological pathways are specially illustrated in the KEGG database, and all KEGG biological pathways can be expanded to and crossed with other pathways (Zhao and Shao, 2009).

Based on the results of the GO analysis, the up-regulated differentially expressed genes were mainly associated with the binding of protein and metal ions (molecular function); these were also correlated to the composition of extracellular matrix, peptidase activity, integration of protein binding, and transcription factor activity. The down-regulated differentially expressed genes were mainly associated with enzyme activity, ion binding and movement, and ion pathway activity. The up-regulated differentially expressed genes were also correlated with several biological processes, such as cell adhesion, collagen fiber formation, decomposition of collagen, cell differentiation, cell signal transduction, cell hypoxia response, cell matrix adhesion, integrin mediated signaling pathway, and nerve skeletal development. The downregulated differentially expressed genes, on the other hand, were mainly related to the cellular redox reaction, cell differentiation, lipid metabolism and synthesis, and inflammatory reaction. The results of pathway analysis revealed that the up-regulated differentially expressed genes were involved in the following pathways: extracellular matrix receptor response, focal adhesion connection, biosynthesis of O-GalNAc chitosan, transforming growth factor- $\beta$ signaling pathway, the interaction between cytokines and their receptors, toll-like receptor signaling pathway, actin cytoskeleton system, cell adhesion molecules, and tumor related signaling pathways. A part of the above-mentioned signaling pathway has been shown to be closely related with the development of pathological scars. In conclusion, we verified the reliability of chip testing; based on the results of data analysis, the unconfirmed signal pathway must be investigated further to elucidate the mechanism of pathological scar formation.

\section{Conflicts of interest}

The authors declare no conflict of interest.

\section{ACKNOWLEDGMENTS}

Research supported by the "Eleven Five" project of the Medical Health Research Fund of the Army (Project \#200626Z000058).

\section{REFERENCES}

Ayari $\mathrm{H}$ and Bricca G (2013). Identification of two genes potentially associated in iron-heme homeostasis in human carotid plaque using microarray analysis. J. Biosci. 38: 311-315.

Chen MR, An G, Liu SL and Wei FC (2012). Gene expression of transforming growth factor beta receptor ii in the epidermis of pathological scar. Zhonghua Shao Shang Za Zhi 28: 278-281.

Ray S, Ju X, Sun H, Finnerty CC, et al. (2013). The IL-6 trans-signaling-STAT3 pathway mediates ECM and cellular 
proliferation in fibroblasts from hypertrophic scar. J. Invest. Dermatol. 133: 1212-1220.

Shi WD, Meng ZQ, Chen Z, Lin JH, et al. (2009). Identification of liver metastasis-related genes in a novel human pancreatic carcinoma cell model by microarray analysis. Cancer Lett. 283: 84-91.

Simpson CD, Anyiwe K and Schimmer AD (2008). Anoikis resistance and tumor metastasis. Cancer Lett. 272: 177-185.

Wang Q (2007). The expression and significance of NEDD4-1 gene in pathological scar. Zhongguoweichuangwaikezazhi 7: 385-387.

Yoshida S and Ishibashi T (2013). Development of novel molecular targeting therapy for diabetic retinopathy based on genome-wide gene expression profiling. Hukuoka acta medica 104: 240-247.

Zhao M and Shao GR (2009). The expression and significance of NEDD4-1 gene in pathological scar. Xiandaishengwuyixuejinzhan 9: 382-384. 